\title{
Uso de juego de roles con grabación de video para el desarrollo de la competencia de entrevistar en estudiantes de psicología
}

\author{
Use of Role Playing With Video Recording to Develop Interviewing Skills in Students of \\ Psychology
}

\section{Uso de dramatização com gravação de vídeo para o desenvolvimento da competência da entrevista em estudantes de psicologia}

Ximena Suárez-Cretton Universidad Arturo Prat Victoria, Chile xsuarez@unap.cl

Carmen Gloria Muñoz-Vilches

Universidad Arturo Prat

Victoria, Chile

carmengm@unap.cl

https://orcid.org/0000-0003-3374-8202

Recibido • Received • Recebido: 23 / 07 / 2018

Corregido • Revised • Revisado: 05 / 12 / 2019

Aceptado • Accepted • Aprovado: 10/ 02 / 2020

Resumen: La investigación es una experiencia de innovación docente a través de una estrategia de simulación, juego de roles, poco usada en la docencia universitaria. El propósito fue verificar si el juego de roles con grabación de video es una estrategia efectiva para la adquisición de la competencia de entrevistar y explorar si este favorece el desarrollo de aprendizaje significativo en el estudiantado. El método consideró una investigación de tipo mixta y un diseño pre-experimental de una sola medición al final. La intervención pedagógica fue realizada en una asignatura de Psicología Laboral durante un periodo de 4 meses. El juego de roles fue la estrategia protagonista. Participaron 13 estudiantes que conformaban el curso. Posterior a la intervención, se realizó una evaluación para estimar el impacto de la experiencia a través de tres instrumentos: Escala de aprendizaje significativo, de Guirao-Goris et al. (2007); Cuestionario de Innovación didáctica, de Bartlett y Morrow (2001), y una Rúbrica para evaluar el logro la competencia. Se analizaron descriptivamente los datos. Los 
http://doi.org/10.15359/ree.24-2.2

ROR: https://ror.org/01t466c14 Universidad Nacional, Costa Rica

http://www.una.ac.cr/educare

educare@una.cr

resultados evidencian, en el estudiantado, una valoración favorable de la estrategia usada para lograr aprendizajes efectivos y una percepción de logro de aprendizaje significativo y, en el docente, una evaluación que avala el logro del aprendizaje de la competencia de entrevista.

Palabras claves: Juego de roles; innovación pedagógica; competencia; aprendizaje; entrevista.

\begin{abstract}
The research is a teaching innovation experience through a simulation strategy, role play, little used in university teaching. The purpose was to verify if role play with video recording is an effective strategy for acquiring interviewing skills, and explore if it helps the development of meaningful learning in students. The method considered a mixed type investigation and a pre-experimental design of a single measurement at the end. The pedagogical intervention was conducted in the Work Psychology course for four months. The main strategy used was role-playing. Thirteen students participated; they were enrolled in this course. After the intervention, to estimate the impact of the experience, an evaluation was made through three instruments: a meaningful learning scale from Guirao-Goris et al. (2007), a questionnaire of didactic innovation from Bartlett y Morrow (2001), and a rubric to evaluate the achievement of the skill. Data were descriptively analyzed. The results show a favorable assessment from the students towards the strategy used to achieve effective learning, perception from the students of significant learning achievement, and an evaluation from the professor endorsing the achievement of learning the interviewing skill.
\end{abstract}

Keywords: Role play; pedagogical innovation; skill; learning; interview.

Resumo: Esta pesquisa é uma experiência de ensino da inovação através de uma estratégia de simulação, dramatização pouco utilizada no ensino universitário. O objetivo foi verificar se a dramatização com gravação de vídeo é uma estratégia eficaz para adquirir a competência de entrevistar, e explorar se favorece o desenvolvimento da aprendizagem significativa nos alunos. $\mathrm{O}$ método considerou uma investigação de tipo misto e um projeto pré experimental de uma única medição no final. A intervenção pedagógica foi realizada em um tema de Psicologia do Trabalho durante um período de 4 meses. A dramatização foi a principal estratégia. Os 13 alunos que compõem o curso participaram. Após a intervenção, foi feita uma avaliação para estimar o impacto da experiência através de três instrumentos: Escala de aprendizagem significativa por Guirao-Goris et al. (2007); Questionário de Inovação didática de Bartlett y Morrow (2001) e uma Rubrica para avaliar a conquista da competição. Os dados foram analisados descritivamente. Os resultados mostram uma avaliação favorável por parte dos alunos da estratégia utilizada para alcançar uma aprendizagem efetiva; uma percepção nos alunos de uma aprendizagem significativa e uma avaliação do professor que endossa a aprendizagem por meio de entrevistas.

Palavras chave: Dramatização; inovação pedagógica; competição; aprendendo; entrevista.

\title{
Introducción
}

La globalización ha presionado porla obtención de mayor calidad en el sistema universitario para poder competir con nuevos estándares. Ello ha generado un cambio de paradigma de aprendizaje: desde uno centrado en la enseñanza, hacia otro, el constructivismo, cuyo centro 
http://doi.org/10.15359/ree.24-2.2

ROR: https://ror.org/01t466c14 Universidad Nacional, Costa Rica http://www.una.ac.cr/educare

es el alumnado. Este plantea la necesidad de basarse en los saberes previos del estudiantado para otorgarle herramientas que le permitan construir sus propios procesos de aprendizaje que les ayuden a resolver situaciones problemáticas (Ortiz, 2015). Por lo tanto, la universidad, en la actualidad, debe favorecer procesos de aprendizaje eficientes en el estudiantado (Gisbert et al., 2010). En esta dirección, la aplicación de estrategias y didácticas orientadas a la obtención de aprendizaje significativo es un aspecto clave que debe estar presente en la formación profesional moderna.

En este contexto, la docencia universitaria se ha visto en la necesidad de evolucionar de contenidos a competencias en coherencia con el mercado laboral, ha sido necesaria la transformación de las estrategias de enseñanza hacia métodos en donde el alumnado sea protagonista y logre adquirir competencias. Actualmente existe una gran cantidad de estrategias de enseñanza a disposición del personal docente, pero se observa que los profesorados universitarios aún muestran desconocimiento y resistencia al cambio de aquellas (Gaete-Quezada, 2011). En una investigación realizada en estudiantes de pedagogía y docentes, los resultados mostraron que el $75 \%$ de los sujetos encuestados nunca había jugado en la universidad, utilizando un rol (Grande de Prado y Abella (2010). Según Moreira (2000), en ocasiones la entrega de contenidos de las asignaturas se realiza en total desconexión de los aprendizajes previos del estudiantado, que son los que permiten el logro de aprendizajes significativos. Moreira (2000) define aprendizaje significativo como "un proceso a través del cual una misma información se relaciona de manera no arbitraria ni literal con un aspecto relevante de la estructura cognitiva del individuo" (p. 10).

La formación en competencias profesionales es otro de los desafíos que la educación superior ha debido incorporar a su nuevo itinerario formativo. El Proyecto Tuning Latinoamericano identifica competencias específicas (propias de cada disciplina) y genéricas (comunes a varias titulaciones) (Beneitone et al., 2007); estas conforman en la educación actual una parte fundamental del perfil profesional de las titulaciones o carreras. Por consiguiente, el desafío para el personal docente está en diseñar metodologías que favorezcan su adquisición en cada programa formativo (de Miguel, 2006).

Desde otra perspectiva, también es importante hacer mención del incremento extraordinario que ha experimentado la matrícula universitaria en los últimos años. Actualmente en Chile el 40 $\%$ de la población entre 18 y 24 años está matriculado en un programa de estudios superiores (Consejo Nacional de Educación [CNED], 2017). Este escenario ha generado cambios tales como: la escasez del recurso de campos clínicos para prácticas en terreno del estudiantado; cambio en los planes desde contenidos a competencias, licitación de los campos clínicos, entre otros.

Trabajar en entornos reales no siempre es posible y, por ello, la educación universitaria requiere proporcionar al estudiantado experiencias lo más cercanas a la realidad para aprender a enfrentar los desafíos que se viven en un entorno clínico real. La utilización de nuevas 
http://doi.org/10.15359/ree.24-2.2

ROR: https://ror.org/01t466c14 Universidad Nacional, Costa Rica

http://www.una.ac.cr/educare

educare@una.cr

herramientas que acerquen al estudiantado a contextos naturales es un desafío ineludible. En consecuencia, se torna determinante disponer de otras instancias que permitan ejercitar las competencias requeridas por el estudiantado; una de estas puede ser los espacios de simulación. “Una simulación podría ser descrita como una representación de una realidad observada o imaginada que obliga a tomar decisiones de acción que tienen efectos con su correspondiente retroalimentación" (Gisbert et al. 2010, p. 365). El role playing o juego de roles corresponde a una estrategia de simulación que habitualmente es utilizada como estrategia pedagógica que aporta beneficios. Favorece el aprendizaje porque permite dedicar más tiempo por parte del estudiantado y mejora la calidad de este al permitir que se otorgue mayor atención a los aspectos más significativos o de interés (Contreras Gelves et al., 2010).

En relación con los antecedentes presentados, el problema de investigación puede resumirse en la siguiente pregunta: ¿es el juego de roles con grabación en video una estrategia efectiva para la adquisición de la competencia de entrevistar y favorece el logro de un aprendizaje significativo?

El objetivo de este estudio es examinar si el juego de roles, con grabación de video, es una estrategia efectiva para la adquisición de la competencia de entrevistar y si favorece el aprendizaje significativo en el estudiantado.

Para lograr este objetivo se propone implementar la estrategia pedagógica de simulación de juego de roles, que ha sido poco usada en docencia universitaria, y observar cómo impacta en el aprendizaje de un grupo de estudiantes.

Los aportes del estudio pueden traducirse en la obtención de evidencia científica que verifique la efectividad de la estrategia pedagógica de juego de roles en el logro de aprendizajes, específicamente competencias; destacar una estrategia didáctica de enseñanza y aprendizaje basada en lo lúdico, que favorezca la adquisición de procedimientos técnicos, y contar con una sistematización de la estrategia de juego de roles para el aprendizaje de competencias específicas y genéricas.

\section{Revisión de antecedentes teóricos}

Gaete-Quezada (2011) plantea que es relevante que el personal docente use estrategias y didácticas innovadoras que lo induzcan a potenciar un ejercicio de una docencia más constructivista que tendrá impacto en el estudiantado.

En los procesos de formación en la universidad, la incorporación del juego de roles es menos frecuente y circunscrita a determinadas formaciones profesionales, a pesar de sus ventajas. 
http://doi.org/10.15359/ree.24-2.2

ROR: https://ror.org/01t466c14 Universidad Nacional, Costa Rica http://www.una.ac.cr/educare

Según Krain y Lantis (2006), los ejercicios de simulación, como el juego de roles, mejoran la experiencia educativa, pues promueven el pensamiento crítico y las habilidades analíticas, el fomento de las competencias de comunicación oral y escrita, y el fomento de la confianza del estudiantado.

El uso de juegos de roles permite comprender y vivenciar la realidad de otras personas; resolver conflictos, reflexionar (García-Barrera, 2015).

En la formación universitaria, el juego de roles es una técnica de aprendizaje activa que proporciona la posibilidad de combinar nuevos conocimientos o información con conocimientos previos (DeNeve y Heppner, 1997). El juego de roles promueve el uso de un enfoque profundo de aprendizaje que exige la interacción y colaboración del estudiantado al favorecer aprendizajes significativos (Schaap, 2005). El uso de un enfoque profundo se refiere a un tipo de procesamiento de la información del estudiantado orientado a comprender el significado del material que debe aprenderse. En contraposición, el uso de un enfoque superficial se dirige a un procesamiento de información orientado en el aprendizaje de la información en sí misma, mediante estrategias más memorísticas (Freiberg y Fernández, 2016). Según los autores, el uso de un enfoque por sobre el otro depende de las acciones de cada docente. En este caso, el uso del juego de roles como estrategia docente favorece un aprendizaje comprensivo propio del aprendizaje significativo.

Las características del juego de roles señaladas serían compatibles con el desarrollo de aprendizajes significativos.

El juego de roles permite analizar los problemas relacionados con las relaciones humanas y fortalece la capacidad de la empatía (Ormazábal et al., 2019). Según Petrizan-Aleman (2016), la estrategia juego de roles"potencia la automotivación en los Juegos de Rol, frente a la enseñanza tradicional. El estudiantado no tiene la sensación de estar estudiando, y las actividades que realizan para cumplir sus tareas se convierten en voluntarias y colaborativas" (p. 24).

En los programas de formación con enfoque por competencias, existen contenidos conceptuales, procedimentales y actitudinales, y el juego de roles posibilita la asimilación de todos estos contenidos (Grande de Prado y Abella, 2010). Porter (2008) también destaca el impacto motivacional que genera para el estudiantado aplicar la estrategia juego de roles y recomienda su uso para el personal docente.

La investigación de Gaete-Quezada (2011) en población universitaria destaca resultados importantes del uso del juego de roles para el aprendizaje, ya que permite una mejor aplicación de los contenidos de clase a situaciones similares al futuro ejercicio profesional; favorece la interacción entre pares y la relación entre teoría y práctica. Estos aspectos son coherentes con el concepto de aprendizaje significativo del paradigma constructivista. 
http://doi.org/10.15359/ree.24-2.2

ROR: https://ror.org/01t466c14 Universidad Nacional, Costa Rica

http://www.una.ac.cr/educare

educare@una.cr

Además, la grabación permite planificar, ensayar y mejorar las propuestas. Los estudiantes tienen la posibilidad de realizar varias tentativas de ensayo en ambientes extra-clase antes de la ejecución final, lo que disminuye el temor que provoca la actuación en vivo y en directo.

Para cumplir el objetivo general, se proponen los siguientes objetivos específicos: a) Diseñar y ejecutar el procedimiento de innovación didáctica con la técnica de juego de roles con grabación audiovisual para la asignatura. b) Verificar si la estrategia utilizada favorece el aprendizaje significativo. c) Evaluar la percepción del estudiantado sobre esta experiencia de innovación docente.

\section{Metodología}

\section{Diseño de investigación}

Se realiza una investigación mixta con un enfoque predominantemente cuantitativo, a través de un diseño pre-experimental, de estudio de caso único de una sola medición después o final. Este consistió en una intervención que aplicaba la estrategia de juego de roles en el que se analiza una asignatura. Para recoger información cualitativa se les agregó a los instrumentos cuantitativos una línea para obtener comentarios u observaciones de estudiantes. La variable central del estudio fue el procedimiento de innovación metodológica: uso de juego de roles con grabación audiovisual. La investigación es descriptiva.

\section{Participantes}

La muestra estuvo conformada por 13 estudiantes de psicología de $8^{\circ}$ semestre de la asignatura de Taller Intervención Laboral de la carrera de psicología, 8 mujeres y 5 hombres, de 21 a 25 años. La selección de la muestra fue intencionada considerando la importancia que tiene la adquisición de la competencia de entrevistar para esta asignatura. Contempló a todo el alumnado del curso. La asignatura utilizada pertenece al área de formación profesional, es práctica y su propósito es favorecer el desarrollo de diferentes competencias relacionadas al área de la psicología laboral tales como: seleccionar personal, capacitar, diagnosticar en el ámbito de las organizaciones o empresas. La intervención se llevó a cabo en el segundo semestre del año 2017, durante 8 semanas en el horario de clases habitual del estudiantado con el propósito de desarrollar la competencia de entrevistar.

\section{Instrumentos}

Se utilizaron tres instrumentos, uno para medir el aprendizaje significativo (cuantitativo); otro para valorar la experiencia de innovación pedagógica con la estrategia de juego de roles (cuantitativo) y luego, una rúbrica (cualitativo) para verificar la adquisición o no de la competencia entrenada: capacidad de entrevistar. 
http://doi.org/10.15359/ree.24-2.2

ROR: https://ror.org/01t466c14 Universidad Nacional, Costa Rica

1. Escala de aprendizaje significativo de Guirao-Goris et al. (2007). Esta versión fue aplicada a estudiantes de enfermería de la Escuela de Enfermería La Fe del primer curso 2007-2008. El instrumento fue aplicado en 59 estudiantes, se obtuvo una consistencia interna $a=$ 0,742. Para explorar la validez de constructo del cuestionario, se realizó un análisis factorial exploratorio y se obtuvo que el instrumento explica un $60.24 \%$ de la varianza, se agrupó en tres dimensiones: aprendizaje significativo (AS) con 6 ítems, aprendizaje cooperativo (AC) con 2 ítems, y recursos (R) con 2 ítems. La escala consta de 10 ítems de respuesta tipo Likert que oscila desde muy en desacuerdo a muy de acuerdo (1 a 5). Se agregó al final de la escala una línea para que el estudiantado escriba comentarios u observaciones de manera libre y abierta.

2. Cuestionario de evaluación de innovación didáctica. Se construyó un cuestionario adhoc para esta investigación, fundamentado en la estructura del cuestionario para evaluar los One minute papers de Bartlett y Morrow (2001). El instrumento se utilizó para que el estudiantado evaluara innovaciones y experiencias didácticas. En esta investigación se utilizó para evaluar la innovación pedagógica con juego de roles. Se conservó la estructura de la prueba, pero se modificó y adaptó el contenido minute paper por estrategia de juego de roles. El cuestionario consta de 10 reactivos. No se considera la presencia de dimensiones. El puntaje oscila de 5 a 50 puntos. Cuanto más alto es el puntaje, más favorable es la actitud. Se obtiene un alfa de Cronbach correspondiente a $a=0.713$; se agregó al final del cuestionario una línea para que el estudiantado escriba comentarios u observaciones de manera libre y abierta.

3. Rúbrica. Instrumento de evaluación de la competencia de entrevista. Fue utilizada con el fin de verificar el nivel de adquisición de la competencia de entrevista. La rúbrica estuvo conformada por la evaluación de 7 criterios (tipo de entrevista, ambiente de entrevista, establecimiento de rapport, guion de preguntas, etc.) con una graduación de 7 categorías de respuesta asociadas también a un puntaje que oscila desde sobresaliente a insatisfactorio.

\section{Procedimientos}

Durante los meses previos a la intervención se diseña la estructura del proceso de innovación a través de juego de roles definiendo las fases y características del proceso, los conocimientos, los roles del estudiantado y de la profesora, los materiales a usar y las formas de evaluación. Esta construcción da origen a un procedimiento sistematizado conformado por tres fases: preparación, entrenamiento y evaluación.

Antes de iniciar la experiencia, el equipo investigador se reunió con el estudiantado para comunicarles sobre la experiencia y conocer su opinión. La experiencia de innovación metodológica a través de juego de roles fue ejecutada durante el segundo semestre del año 
http://doi.org/10.15359/ree.24-2.2

ROR: https://ror.org/01t466c14 Universidad Nacional, Costa Rica

http://www.una.ac.cr/educare

educare@una.cr

2017, durante un periodo de cuatro meses. La intervención fue realizada por la docente titular de la asignatura y monitoreada por el equipo de investigación. Se le comunicaron al estudiantado las características de la intervención y sus objetivos. Posteriormente se solicitó al alumnado la firma de un consentimiento informado en que se resguarda la voluntariedad y confidencialidad de su participación en la experiencia con fines de investigación y se autoriza la aplicación de instrumentos de recolección de datos.

Al término del semestre se llevó a cabo la aplicación de los instrumentos Escala de aprendizaje significativo y Cuestionario de evaluación de la innovación didáctica. Paralelamente, la docente a cargo de la asignatura evalúo individualmente el desarrollo de la competencia obtenida a través de una rúbrica. Posterior, se tabularon los datos y se procedió al análisis. En el análisis de los datos se aplicaron estadísticos descriptivos: medias, frecuencias y desviaciones estándar. Se consideran los puntajes superiores a tres como indicativos de categoría favorable o tendencia a estar de acuerdo, como criterios de análisis de las Escalas de aprendizaje significativo y del Cuestionario de innovación didáctica. Para el caso de la rúbrica, que evalúa el logro de la competencia de entrevistar, se considera un puntaje sobre 4 como indicador de un desempeño satisfactorio. El análisis estadístico se realizó a través del programa SPSS versión 22.

\section{Resultados}

\section{Diseño y ejecución de intervención}

\section{Plan de intervención}

La propuesta fue la aplicación de la técnica de juego de roles con grabación audiovisual para la adquisición de la destreza de entrevista psicológica por parte del alumnado. Se diseñó la siguiente metodología para realizar la experiencia de intervención (Ver figura 1):

Figura 1. Proceso de intervención con técnica juego de roles
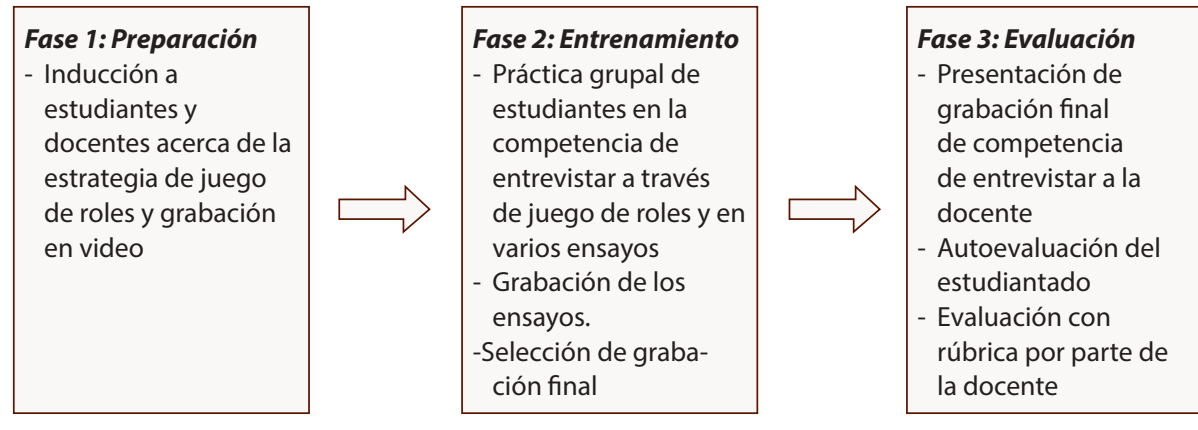

Nota: Elaboración propia 


\section{Fases de la intervención con juego de roles}

\section{Fase 1. Preparación}

Se entregan todas las orientaciones e información al alumnado con respecto a la utilización de la técnica de juego de roles. La docente debe recibir instrucción sobre el juego de roles y su uso en caso de que no lo maneje. Se debe revisar que se cuente con todos los recursos necesarios: cámara de video, salas, pauta de trabajo, temática o caso.

- Revisión de conceptos relativos al tema de entrevista (entorno, preparación, etc.)

- Modelamiento de una entrevista de selección de personal por parte de la docente

- Conformación de parejas para el juego de roles

- Observación del juego de roles de compañeros y compañeras

- Ejercitación de juego de roles y retroalimentación (pares y docente)

\section{Fase 2. Entrenamiento}

Una vez concluida la fase anterior, se debe firmar el juego de roles.

- Ejecución del juego de roles con grabación y retroalimentación en el proceso (autoevaluación y evaluación de la docente)

- Creación de versión final de video de entrevista para evaluación

\section{Fase 3. Evaluación}

Una vez concluida la versión final del juego de roles se procede a la fase de evaluación del desempeño del estudiantado:

- Evaluación de la grabación en video de la entrevista por parte de la docente usando una rúbrica.

- Retroalimentación posterior al estudiantado sobre su desempeño en la competencia de entrevistar.

Una vez concluida la intervención, se ejecutó el proceso de evaluación del impacto de uso de la técnica de juego de roles con grabación a través de los dos instrumentos ya descritos. 
http://doi.org/10.15359/ree.24-2.2

ROR: https://ror.org/01t466c14 Universidad Nacional, Costa Rica

http://www.una.ac.cr/educare

educare@una.cr

\section{Resultados de aprendizaje significativo}

Los resultados obtenidos a través de la percepción del estudiantado verifican la presencia de un aprendizaje significativo que se refleja en la categoría de acuerdo a muy de acuerdo (Ver Tabla 1). Se muestran algunas opiniones del alumnado escritas en la línea para comentarios ubicada al final de la escala. En la dimensión aprendizaje significativo, se observa una percepción favorable (de acuerdo a muy de acuerdo) la cual muestra que la metodología de juego de roles con grabación les permitió desarrollar sus conocimientos de manera más perdurable con base en la articulación con lo que ya sabían, por lo tanto se consiguieron los objetivos planteados en la asignatura y se obtuvo una mayor comprensión en el proceso. Respecto del aprendizaje colaborativo, también se obtuvo una percepción favorable (de acuerdo a muy de acuerdo) y se observa que el desarrollo del conocimiento se logró a través de la discusión y la coordinación con sus pares. En el subdimensión recursos la valoración es favorable (de acuerdo con muy de acuerdo), lo que reconoce los apoyos, materiales, organización y sistematización constante de la profesora (ver Tabla 1).

Tabla 1: Medias de aprendizaje significativo

\begin{tabular}{lccccc}
\hline & N & Mínimo & Máximo & Media & Desviación estándar \\
\hline Apr_Signif & 13 & 4 & 5 & 4,50 & 0,549 \\
Apr_Colab & 13 & 4 & 5 & 4,53 & 0,499 \\
Recursos & 13 & 4 & 5 & 4,61 & 0,487 \\
Apr_Signif Total & 13 & 4 & 5 & 4,551 & 0,048 \\
N válido (por lista) & 13 & & & & \\
\hline
\end{tabular}

Nota: Elaboración propia.

Un análisis con más detalle de la Tabla 1 permite observar que la subdimensión de recursos logra un puntaje más alto que las otras subdimensiones, lo cual refleja la relevancia que tiene para el estudiantado la guía o directriz de la profesora que acompaña la estrategia. Como también la valoración de la técnica como un recurso de aprendizaje adecuado en el logro de la competencia de entrevistar.

A continuación, se agregan algunos comentarios formulados por el estudiantado:

\section{-Subdimensión aprendizaje significativo:}

La utilización del Role Playing es una estrategia que favorece el aprendizaje es más didáctico y mucho más fácil de practicar que los power point. (Participante 2) 
http://doi.org/10.15359/ree.24-2.2

ROR: https://ror.org/01t466c14 Universidad Nacional, Costa Rica

http://www.una.ac.cr/educare

educare@una.cr

Mi comentario es totalmente positivo ya que es una forma muy adecuada de aprendizaje e innovadora lo que también es una buena forma de evaluar y retroalimentar las habilidades como futuro profesional. (Participante 4)

Permite desarrollar nuestro conocimiento teórico que se entrega en la clase en el ámbito más práctico para así enfrentarnos con más seguridad a futuro. (Participante 12)

\section{-Sub-dimensión aprendizaje colaborativo:}

Permite retroalimentación grupal y nos aproxima a una experiencia real. (Participante 6).

Aprendí de forma diferente y en conjunto con mis compañeros algo valioso e innovador. (Participante 7).

\section{-Sub-dimensión recursos:}

Es un método de aprendizaje muy significativo que se consolida en nuestro qué hacer, más aún cuando uno tiene profesores guías que favorecen este tipo de conocimientos. (Participante 11)

La grabación después de revisarla ayuda mucho para darme cuenta lo que me faltó, cómo puedo mejorar y así la próxima entrevista hacerlo de mejor manera e integrar lo que me faltó. (Participante 5)

Los comentarios formulados por el estudiantado corresponden a información cualitativa obtenida en el espacio que se agrega para expresarse espontáneamente. Si bien no corresponden al objetivo formal evaluado por la escala, proporcionan información valiosa sobre los beneficios que conlleva el uso de la estrategia de juego de roles. Los comentarios en general son favorables y diversos con respecto a las temáticas mencionadas. La mayoría de ellos se orienta al uso de competencias genéricas como: colaboración, comunicación, empatía, entre otras. No obstante, también se refieren a habilidades cognitivas como: autocrítica, evaluación, reflexión, y a aspectos motivacionales como: interés por la tarea, diversión, satisfacción.

\section{Evaluación de la experiencia de innovación}

La experiencia fue valorada favorablemente por el estudiantado en cada uno de los ítems que conformó el instrumento y mostró que esta metodología mejoró la adquisición de la competencia para entrevistar y el aprendizaje de procedimientos. Además, revela que resultó útil observar a otros sujetos al realizar la tarea, así como recibir retroalimentación de sus compañeros y compañeras respecto de su ejecución. Fue valorada positivamente como una 
http://doi.org/10.15359/ree.24-2.2

ROR: https://ror.org/01t466c14 Universidad Nacional, Costa Rica

http://www.una.ac.cr/educare

educare@una.cr

estrategia para ser usada también en otras asignaturas y resultó importante para el desarrollo de la competencia de entrevista $(M=4,40 D S=0,641)$.

Algunas opiniones del estudiantado:

Me sirvió para fortalecer habilidades y sobre todo para aplicar de manera práctica los contenidos. (Participante 2)

El juego de roles es de gran utilidad puesto que es una estrategia que facilita el aprendizaje. (Participante 12)

Una experiencia nueva, difícil y de gran aprendizaje. (Participante 3)

El aprendizaje es más experiencial... nos acerca a los escenarios de una entrevista real. (Participante 5)

\section{Resultados obtenidos en la rúbrica aplicada por la docente para evaluar la adquisición de la competencia}

Los resultados obtenidos en la rúbrica verifican la obtención de las categorías de bueno a muy bueno en la mayoría de los criterios evaluados. Solo en el criterio aplicación de la teoría el estudiantado obtiene un desempeño más bajo correspondiente a la categoría de regular a bueno. Donde obtienen un mejor desempeño es en la categoría participación del grupo logrando una media de 6,7 (Ver Tabla 2).

Tabla 2: Distribución de medias del instrumento de evaluación rúbrica

\begin{tabular}{lccccc}
\hline & N & Mínimo & Máximo & Media & Desviación estándar \\
\hline Tipo_entrevista & 12 & 5,00 & 7,00 & 6,0833 &, 79296 \\
Amb_entrevista & 12 & 5,00 & 7,00 & 6,5833 &, 79296 \\
Rapport & 12 & 5,00 & 7,00 & 5,8333 &, 83485 \\
Guión_preguntas & 12 & 5,00 & 7,00 & 5,7500 &, 62158 \\
Consig_resp & 12 & 5,00 & 7,00 & 5,7500 &, 62158 \\
Aplic_teorías & 12 & 4,00 & 6,00 & 4,9167 &, 51493 \\
Partic_grupo & 12 & 6,00 & 7,00 & 6,7500 &, 45227 \\
\hline
\end{tabular}

Nota: Elaboración propia. 
http://doi.org/10.15359/ree.24-2.2

ROR: https://ror.org/01t466c14 Universidad Nacional, Costa Rica

Los resultados obtenidos en la rúbrica indican que el aprendizaje de la competencia de entrevista fue logrado en un grado de bueno a muy bueno. Esto significa que el estudiantado desarrolló la competencia de entrevista.

\section{Discusión}

Como resultado de la intervención realizada en la muestra de estudiantes de psicología con la estrategia de juego de roles con grabación de video, se obtuvieron los siguientes resultados:

La utilización de la estrategia juego de roles con grabación es una estrategia efectiva para el desarrollo de la competencia de entrevistar en estudiantes en formación de la carrera de psicología. Según la percepción de estudiantes, la estrategia permitiría un aprendizaje significativo que logra relacionar, de mejor forma, la nueva información con sus conocimientos previos. El alumnado construyó su conocimiento discutiendo y debatiendo con otros sujetos, orientándose al objetivo planteado por la docente, organizando su tiempo para trabajar de manera autónoma, realizando varios intentos de aplicación de la técnica que contribuyó de forma paulatina a construir el conocimiento sobre la competencia de entrevistar.

El constructivismo plantea la necesidad de basarse en los saberes previos del estudiantado para otorgarle herramientas que le permitan construir sus propios procesos de aprendizaje con la finalidad de resolver situaciones problemáticas (Moreira, 2000; Ortiz, 2015). La práctica constructivista implica que el estudiantado debe aprender de la experiencia y no solo de la lectura y análisis teórico. Se trata de un aprender haciendo a través de la experimentación. En este sentido, la técnica de juego de roles es coherente con los requerimientos del modelo constructivismo, dado que desafía al estudiantado a adoptar una actitud activa hacia su proceso de aprendizaje.

A continuación, se analizan los objetivos específicos del estudio con el fin profundizar en los resultados:

En primer lugar, la finalidad de la investigación fue innovar en la docencia universitaria probando una estrategia de uso menos frecuente para el logro de aprendizaje significativo. Para el desarrollo de este objetivo, la investigación sistematizó un procedimiento de intervención usando la técnica de juego de roles de modo que puede ser utilizada en cualquier cátedra para la adquisición de contenidos de tipo procedimental. En ella se describen las distintas fases que deben llevarse a cabo con las personas participantes. Estas son preparación, entrenamiento y evaluación. La sistematización realizada del procedimiento se considera un producto que aporta en la innovación docente en educación superior al proporcionar un método que permite generar en el estudiantado un aprendizaje útil, el cual fue probado en una muestra de estudiantes. En términos generales, la ejecución fue llevada a cabo de acuerdo con lo programado, pues no se presentaron situaciones imprevistas o no consideradas. Una de las dificultades que evidencia 
http://doi.org/10.15359/ree.24-2.2

ROR: https://ror.org/01t466c14 Universidad Nacional, Costa Rica

http://www.una.ac.cr/educare

educare@una.cr

la utilización de la técnica es el uso de una cantidad de tiempo mayor al de otras estrategias pedagógicas tanto para el estudiantado como para el personal docente.

Luego se propuso evaluar si esta metodología promueve la adquisición de aprendizaje significativo. Los resultados obtenidos estiman que la técnica de juego de roles utilizada fue apropiada para el logro de aprendizaje significativo, de acuerdo con el autorreporte realizado por el estudiantado participante, donde se refleja una actitud de muy de acuerdo en la escala total y en los subdimensiones evaluadas. El estudiantado percibió el logro de un aprendizaje significativo, útil, que le permitió relacionar lo antiguo con lo nuevo, lo teórico con lo práctico, en el que sintieron comodidad e interés. Como un hallazgo adicional resultante de la información cualitativa recolectada por el instrumento, en el espacio comentarios, el estudiantado plantea percibir la adquisición de competencias genéricas como: comunicación, pensamiento crítico, trabajo colaborativo, entre otras. Este resultado es concordante con lo propuesto por DeNeve y Heppner (1997) sobre el juego de roles como una técnica activa que promueve un aprendizaje significativo y con la propuesta de Schaap (2005) referida al uso de un enfoque profundo de aprendizaje. La bibliografía señala la conveniencia de desarrollar aprendizajes orientados al uso de un enfoque profundo para el logro de aprendizajes significativos, es decir, seleccionar estrategias que favorezcan en el estudiantado un procesamiento de la información centrado en la comprensión, más que en la memorización. La investigación de Krain y Lantis (2006) destaca la promoción del pensamiento crítico y de las habilidades analíticas como consecuencia del uso del juego de roles. El estudiantado participante a través de comentarios espontáneos menciona como fortalezas la adquisición de habilidades similares: La grabación después de revisarla ayuda mucho para darme cuenta lo que me faltó (Participante 5).

En la subdimensión de aprendizaje colaborativo del mismo instrumento también se obtuvo una percepción favorable respecto al desarrollo del conocimiento logrado a través de la discusión y la coordinación con sus pares. Esta idea es apoyada por Ormazabal et al. (2019) y por Grande de Prado y Abella (2010) al concordar que la utilización de juegos de roles permite comprender y vivenciar la realidad de otros seres a través de la empatía, mejora la comunicación y la tolerancia hacia la diversidad.

Lo mismo ocurre en la subdimensión recursos en que se valora la importancia de los materiales, lo técnico y humano. El estudiantado identifica como relevante el aporte que realiza la docente a cargo de la asignatura en la planificación, organización, sistematización y evaluación, como también el atractivo e interés que la técnica genera. Este resultado es coincidente con lo plateado por Gaete-Quezada (2011) cuando sugiere que el personal docente use estrategias y didácticas innovadoras como el juego de roles, que induzcan a potenciar un ejercicio de una docencia más constructivista que tendrá impacto en el estudiantado.

A la luz de los resultados obtenidos se confirma el aporte de la estrategia de juego de roles hacia la obtención de aprendizajes útiles que, según la percepción del estudiantado, 
http://doi.org/10.15359/ree.24-2.2

ROR: https://ror.org/01t466c14 Universidad Nacional, Costa Rica

es un aprendizaje significativo. Como también la sitúa como una estrategia efectiva para la adquisición de contenidos procedimentales, y de actitudes y valores propias de un aprendizaje integral. Al mismo tiempo, puede ser calificada como una estrategia o técnica pedagógica efectiva para la adquisición de la competencia de entrevistar y, probablemente, también para otras competencias profesionales con un fuerte componente práctico.

Con respecto a la valoración que realiza el estudiantado de la experiencia de innovación, nuevamente se obtiene una valoración muy positiva que se refleja en actitudes muy favorables en todos los aspectos explorados. Según Petrizan-Aleman (2016, p. 24), cuando estudiantes usan el juego de roles "no tienen la sensación de estar estudiando, y las actividades que realizan para cumplir sus tareas se convierten en voluntarias y colaborativas". Este planteamiento va en la misma dirección con la evaluación reportada en este estudio, en la cual se destaca el aumento de la motivación y del interés por la tarea. Petrizan-Aleman (2016) destaca el potencial de automotivación que se asocia al uso de juegos de roles. Por ejemplo: Aprendí de forma diferente y en conjunto con mis compañeros algo valioso e innovador (Participante 7).

También es importante destacar que el uso de juego de roles en la intervención fue utilizado como una estrategia metodológica para estimular el desarrollo de competencias en este caso entrevistar; pero, además, como un instrumento de evaluación. En la valoración de la experiencia de innovación el estudiantado se refiere positivamente al uso del juego de roles como estrategia de evaluación. En las opiniones del alumnado se valora favorablemente el potencial de retroalimentación que surge espontáneamente de la experiencia tanto a nivel de autoevaluación y coevaluación de pares y de la docente. Afirman que evaluar a través de una técnica como esta u otra es mucho más útil y significativo que la utilización de evaluaciones tradicionales, relacionadas con repetición y memorización de contenidos y conceptos. Un aspecto de la intervención que podría considerarse como una debilidad o aspecto a mejorar es el uso del tiempo. La estrategia requiere de una planificación y organización previa para el profesorado, como también, de una cantidad de horas para su ejecución. Una parte de estudiantes manifiesta que ocupa mucho tiempo, que es difícil y que puede ser vivenciada con temor frente a la propia autoevaluación como a la evaluación de otras personas. Sin embargo, tal como lo platea Gaete-Quezada (2011), el uso de grabación audiovisual (video) disminuye el miedo que genera la actuación «en vivo y en directo» y permite la corrección de errores no siempre perceptibles en actuaciones en vivo.

Se incorporó también en el estudio la evaluación formal que realizó la docente a cargo de la asignatura para verificar si el estudiantado adquirió la competencia de entrevista. Esta evaluación se realizó a través de una rúbrica que fue completada por la docente tras la exhibición de los videos realizados por el estudiantado. Los resultados obtenidos indican el logro de la competencia en un grado de bueno a muy bueno para la mayoría de las personas participantes. Este resultado evidencia que la estrategia sirvió para desarrollar la competencia de entrevistar, 
http://doi.org/10.15359/ree.24-2.2

ROR: https://ror.org/01t466c14 Universidad Nacional, Costa Rica

http://www.una.ac.cr/educare

educare@una.cr

resultado que es coincidente, además, con la percepción de aprendizaje significativo y con la valoración de la innovación por el estudiantado. No obstante, lo anterior, en el instrumento usado se refleja un rendimiento más bajo en el dominio conceptual de la competencia. Una posible explicación podría ser que el personal docente no enfatice la importancia de la teoría para el desarrollo del ejercicio de juego de roles. Desde el punto de vista estudiantil podría denotar una dificultad en el grupo para relacionar la teoría con la práctica. Sin duda, este aspecto debe ser considerado por los cuerpos docentes cuando se utiliza la técnica de juego de roles para la obtención de resultados efectivos.

La técnica de juego de roles puede constituir un buen método para lograr el desarrollo de las competencias requeridas por el medio laboral actual (Beneitone et al., 2007). Parece fundamental trabajar en el aula con metodologías apropiadas para entrenar competencias (de Miguel, 2006), el juego de roles puede ser una de ellas.

\section{Conclusiones}

En síntesis, es posible afirmar que el uso de la técnica juego de roles es muy recomendable para la enseñanza de la competencia de entrevistar y genera, en el estudiantado, la percepción de logro de aprendizajes significativos. Como un hallazgo adicional, reflejado en los comentarios planteados por estudiantes, se constata que la técnica de juego de roles fortalece el desarrollo de competencias genéricas como: comunicación, habilidades de cooperación, aumento de la motivación, entre otras.

El aporte de la investigación se traduce en poner a disposición de docentes una estrategia pedagógica innovadora al servicio del desarrollo y evaluación de competencias específicas y genéricas, al incorporar estrategias didácticas vivenciales. Dentro de sus ventajas, permite la construcción de material (videos) que pueden ser usados por otros grupos de estudiantes, favorece que el proceso de aprendizaje sea personalizado, pues se adapta a las características de cada estudiante. Es una técnica de evaluación asincrónica, de manera que el personal docente pueda analizarla o evaluarla en tiempos distintos a su realización. No requiere de altos costos.

La investigación no está exenta de limitaciones. La experiencia se realiza en una muestra pequeña seleccionada intencionadamente y correspondiente a una carrera específica. Evaluar el aprendizaje significativo es muy difícil, dado que este puede ocurrir en diferentes tiempos; por ello se incorpora la percepción de aprendizaje significativo. Los resultados obtenidos pueden depender bastante de las características del grupo y su efectividad es variable, pues depende de la madurez del grupo curso. 
http://doi.org/10.15359/ree.24-2.2

ROR: https://ror.org/01t466c14 Universidad Nacional, Costa Rica

\section{Referencias}

Bartlett, M. G. y Morrow, K. A. (2001). Method for assessing course knowledge in a large classroom environment: An improved version of the minute paper. American Journal of Pharmaceutical Education, 65, 264-267. http://citeseerx.ist.psu.edu/viewdoc/download?d oi=10.1.1.623.3680\&rep=rep1\&type $=$ pdf

Beneitone, P., Esquetini, C., González, J., Marty, M, Siufi, G. y Wagenaar, R. (Eds.). (2007). Reflexiones y perspectivas de la educación superior en América Latina. Informe final -Proyecto Tuning- América Latina 2004-2007. Universidad de Deusto. http://tuningacademy.org/ wp-content/uploads/2014/02/TuningLAllI Final-Report SP.pdf

Consejo Nacional de Educación (CNED). (2017). Índices tendencias educación superior 2017. https://www.cned.cl/sites/default/files/tendencias matricula pregrado 2017.pdf

Contreras Gelves, G., García Torres, R. y Ramírez Montoya, M. S. (2010). Uso de simuladores como recurso digital para la transferencia de conocimiento. Apertura, 2(1), 1-47. http://www. udgvirtual.udg.mx/apertura/index.php/apertura/article/view/22/33

de Miguel, M. (dir.) (2006). Modalidades de enseñanza centradas en el desarrollo de competencias. Orientaciones para promover el cambio metológico en el espacio europeo de educación superior. Servicio de Publicaciones de la Universidad. https://www2.ulpgc.es/hege/ almacen/download/42/42376/modalidades ensenanza competencias mario miguel2 documento.pdf

DeNeve, K. M. y Heppner, M. J. (1997). Role play simulations: The assessment of an active learning technique and comparisons with traditional lectures. Innovative Higher Education, 21(3), 231-246. https://doi.org/10.1007/BF01243718

Freiberg Hoffman, A. y Fernández Liporace, M. M. (2016). Enfoques de aprendizaje en estudiantes universitarios argentinos según el R-SPQ-2F: Análisis de sus propiedades psicométricas. Revista Colombiana de Psicología, 25(2), 307-329. https://doi.org/10.15446/ rcp.v25n2.51874

Gaete-Quezada, R. A. (2011). El juego de roles como estrategia de evaluación de aprendizajes universitarios. Educación y Educadores, 14(2), 289-307. Recuperado de http://www.redalyc. org/articulo.oa?id $=83421404005$

García-Barrera A. (2015). Importancia de la competencia argumentativa en el ámbito educativo: Una propuesta para su enseñanza a través del role playing online. Revista de Educación a Distancia, 45, 1-20. https://doi.org/10.6018/red/45/alba 
http://doi.org/10.15359/ree.24-2.2

ROR: https://ror.org/01t466c14 Universidad Nacional, Costa Rica

http://www.una.ac.cr/educare

educare@una.cr

Gisbert, M., Cela-Ranilla J. M. e Isus, S. (2010). Las simulaciones en entornos tic como herramienta para la formación en competencias transversales de los estudiantes universitarios. TESI Teoría de la educación y cultura de la Sociedad de la Información, 11(1), 352-370. http:// revistas.usal.es/index.php/eks/article/view/6309/6322

Grande de Prado, M. y Abella, V. (2010). Los juegos de rol en el aula. TESI Teoría de la educación y cultura de la Sociedad de la Información, 11(3), 56-84. http://www.redalyc.org/ pdf/2010/201021093004.pdf

Guirao-Goris, S. J. A., Ferrer, E. y Olmedo, A. (2007). Escala par la medición de aprendizaje significativo en alumnos de enfermería [Cartel] Escola Universitària d'Infermeria, Universidad de Valencia. 10.13140/RG.2.1.4426.7040

Krain, M. y Lantis, J. S. (2006). Building knowledge? Evaluating the effectiveness of the global problems summit simulation. International Studies Perspective Journal, 7(4), 395-407. https://doi.org/10.1111/j.1528-3585.2006.00261.x

Moreira, M. A. (2000). Aprendizaje significativo: Teoría y práctica. Aprendizaje Visor.

Ormazábal, V., Almuna, F., Hernández, L. y Zuñiga, F. (2019). Juego de roles como método de enseñanza de farmacología para estudiantes de la carrera de enfermería. Educación Médica, 20(4), 206-212 https://doi.org/10.1016/j.edumed.2018.07.001

Ortiz, D. (2015). El constructivismo como teoría y método de enseñanza. Revista Sophia. Colección de filosofía de la educación, 19, 93-110. https://doi.org/10.17163/soph.n19.2015.04

Petrizan-Aleman, O. (2016). Juego de rol aplicado a la enseñanza de "Mercado de trabajo" en Economía de $1^{\circ}$ de Bachillerato (Tesis de maestría). Universidad Internacional de la Rioja, Pamplona, Navarra. https://reunir.unir.net/handle/123456789/3946

Porter,A.L.(2008). Role-playing and religion:Using gamestoeducatemillennial.Teaching Theology and Religion Journal, 11(4), 230-235. https://doi.org/10.1111/j.1467-9647.2008.00468.x

Schaap, A. (2005). Learning political theory by role-playing. Politics, 25(1), 46-52. https://doi. org/10.1111/j.1467-9256.2005.00228.x 